\title{
HADRON SPECTRUM WITH STAGGERED DYNAMICAL QUARKS*
}

Khalil M. BITAR, A. D. KENNEDY, Weiqiang LIU

SCRI, The Florida State University, Tallahassee, FL 32306-4052, USA

Thomas A. DeGRAND

Physics Department, University of Colorado, Boulder, CO 80309, USA

Steven GOTTLIEB

Indiana University, Bloomington, IN 47405, USA

J. B. KOGUT, R. L. RENKEN

Department of Physics, University of Ilinois, 1110 W. Green Street, Urbana, IL 61801, USA

Michael C. OGILVIE

Department of Physics, Washington University, St. Louis, MO 63130, USA

Pietro ROSSI

Department of Physics, University of California at San Diego, La Jolla, CA 92093, USA

D. K. SINCLAIR

362-HEP, Argonne National Laboratory, Argonne, IL 60439-4815, USA

R. L. SUGAR

Departmen ff Physics, University of California, Santa Barbara, CA 93106, USA

Michael TEPER

Department of Theoretical Physics, University of Oxford, Oxford OXI 3NP, UK

D. TOUSSAINT

Department of Physics, U1.iversity of Arizona, Tucson, AZ 85721, USA

\begin{abstract}
We describe a recent calculation of the hadron spectrum with two flavors of staggered dynamical quarks with a gauge coupling $6 / g^{2}=5.60$ and quark masses of 0.025 and 0.01 . The gauge fields were generated using the hybrid algorithm on a $12^{4}$ lattice that was doubled or quadrupled to calculate hadron propagators.
\end{abstract}

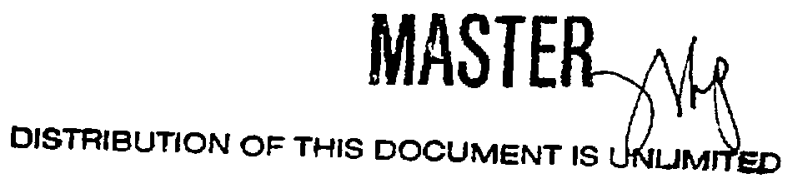

* Presented by Weiqiang Liu at the International Symposium LATTICE '89, CAPRI, Italy, 1989.

Work supported by the U.S. Department of Energy, Division of High Energy Physics, Contract W-31-109-ENG-38. 


\section{INTRODUCTION}

The U.S. Department of Energy is supporting three large supercomputer projects in lattice gauge theory. Two, done on Cray computers are devoted to large quenched calculations. ${ }^{1}$ The third, known as the High Energy Monte Carlo Grand Challenge (HEMCGC), uses the ETA10 at the Sipercomputer Computations Research Institute and is devoted to dynamical fermion calculations.

To make an accurate evaluation of the hadron mass spectrum we must push present calculations to smaller quark masses, smaller lattice spacings and larger volumes. As a step in this direction we have carried out a simulation using two flavors of staggered fermions on $12^{4}$ lattices, and then doubled and quadrupled the lattices to calculate hadron propagators. We studied quark masses $a m_{q}=0.025$ and 0.01 with $6 / g^{2}=5.6$ using the hybrid algorithm. ${ }^{2}$

We use the configurations thus generated to extract a number of interesting physical quantities. While generating the configurations we measured the plaquette, $\psi \psi$ and the Polyakov loop. We later analyzed the configurations to calculate the spectrum. We have used both staggered and Wilson valence quarks for this purpose. Here we concentrate on the staggered spectrum. Khalil Bitar will present the results on the Wilson spectrum. ${ }^{3}$ Future work includes a study of the glueball spectrum and various hadron wave functions and matrix elements.

\section{CODE DEVELOPMENT}

Considerable effort was expended to develop a code that runs efficiently on the ETA10 and can also be debugged on smaller machines. The code is written in Ratfor, anci a macro preprocessor is extensively used.

A stack allocator that has several useful features was created for the program. A large common block is created at compilation time; during run time, pieces of the common block are allocated to temporary variables such as the gauge fields, fermion fields, temporary vectors for the conjugate gradient, etc. A set of macros is used to relate the temporary variables to the appropriate area of the common block. This results in code that makes efficient use of memory and does not require recompilation when changing lattice size (up to a maximum determined by the common block declaration). The resulting code requires about $1.3 \times 10^{-6}$ seconds per lattice site per conjugate gradient iteration for a program that fits in the $4 \mathrm{MW}$ CPU memory.

The ETA10 has a large shared memory; however, the software overhead for using it is high. On a $14^{2} \times 24^{2}$ lattice we found that during the conjugate gradient the ratio of system time to CPU time was 4.5. By time "slicing" the program and manually overlapping the computations and I/O between shared and CPU memories, this ratio was reduced to 0.2 . Each time-sliced routine requires a careful analysis to manage the data movement and allocation.

\section{CALCULATIONAL DETAILS}

After equilibrating a $12^{4}$ lattice, we tested the algorithm by varying the molecular dynamics time step $d t$ and the conjugate gradient accuracy $\epsilon=\sqrt{R^{2} / S^{2}}$ where $R$ is the residual vector and $S$ is the source vector. We chose $d t=0.01(0.02)$ for the light (heavy) mass and $\epsilon=5 \times 10^{-4}$ for production runs. The trajectory length (time 
between refreshing the momenta) is 1 time unit. For the quark masses 0.025 and 0.01 we made runs of 5000 trajectories saving the lattice every 10 time units. Thus, we have 500 configurations for each mass.

To calculate the spectrum, we doubled or quadrupled the lattice in the time direction. The propagators used for the hadrons all employed local sinks, summed over space to obtain zero momentum states. However, we have used both point and wall sources. The wall source is created by placing a unit source on every site for which each of the spatial coordinates is even for a fixed time. When using the wall source, we transformed to Coulomb gauge using an overrelaxation algorithm.

\section{RESULTS}

We studied two $\pi$ propagators, two $\rho$ propagators, and one $\sigma, A_{1}, B$ and nucleon propagator. We used doubled lattices with point sources and with wall sources. For the wall sources, we used both antiperiodic and open boundary conditions in time. We also used quadrupled lattices with a wall source, but only for the lighter quark mass. We fitted the propagators using the full covariance matrix. Details of the fitting procedure may be found in Ref. 4. To extract the long distance fall-off of the hadron propagators we fit from some minimum distance $D_{\min }$ to the center of the lattice on the doubled lattices. In Fig. 1 we show the $\pi$ mass as a function of $D_{\min }$ for the doubled lattices. Using both point and wall sources. For each source, we use two parameter (one particle) fits and four parameter fits that include a $\pi$ and an excited state of the same spin and parity. Looking at the two parameter fits, we see that the point source suffers from considerable contamination up to a distance of four or five from the source, the wall source does not have this problem. When we include an excited state, the poi...t source $\pi$ masses are much more stable. We use the full covariance matrix of the propagators, so the fits' confidence levels are meaningful. An arrow points at each fit with confidence level greater than 0.1 .

On the quadrupled lattices, we varied the maximum distance, generally in the range from 12 to 16 . In Fig. 2 we show the $\pi$ fits on the quadrupled lattice. Because we only have a wall source here we can show all fits on a much finer scale. We see a tendency for the fits with longer maximum distance from the source to give lower masses, this is somewhat disturbing, and we hope to see if it is an artifact of periodic extension in future work. We report masses from two parameter wall source fits on the doubled lattices with $D_{\min }=5$.

Turning to the $\rho$ fits in Fig. 3, we see that the point source again suffers from contamination from higher mass states. There is the familiar pattern of two parameter fits oscillating in mass because of the contribution of the opposite parity state that is ignored in the fit. For the four parameter fits, the oscillation disappears, but the contribution of an excited state with the same quantum numbers as the $\rho$ causes the fitted $\rho$ mass to increase for small $D_{\min }$. The wall source avoids much of the contribution of excited states, but we see the oscillation of the mass in the two parameter fits as for the point sources. The four parameter fits have a reasonable confidence level for $D_{\min } \geq 2$. In Fig. 3(b) we see a similar pattern emerging for the light quark mass. However, the four parameter fits for the wall source do not have a good confidence level.

For the nucleon, the wall source gives much better fits than the point source. The masses from the point source are considerably lighter than those from the wall source. Given the well known problems of using point sources as the coupling decreases, we feel justified in ignoring those fits in this brief summary. In addition, for $a m_{q}=0.01$ the wall source on the quadrupled lattice is in good agreement with that on the 

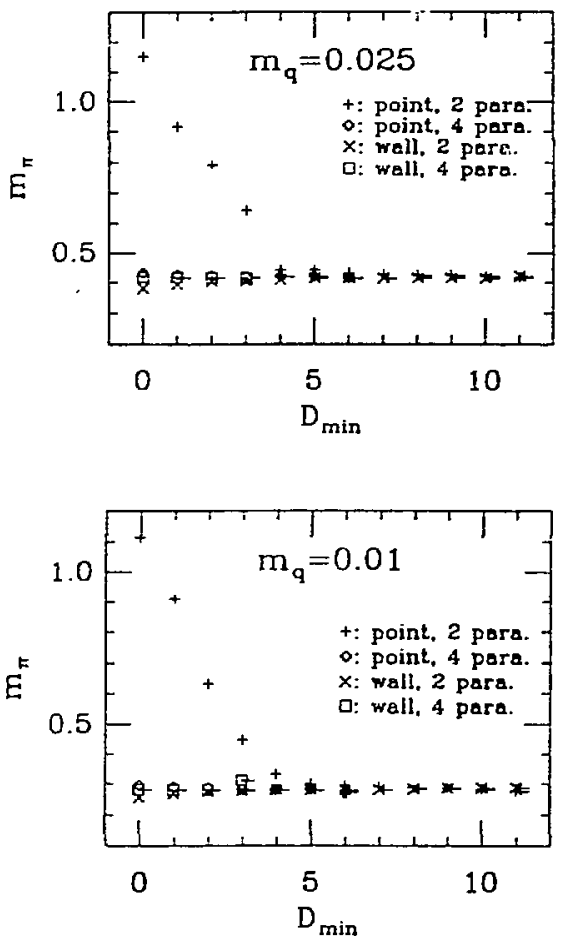

Figure 1. Pion mass for the doubled lattice as a function of the minimum distance from the source included in the fit. (a) $a m_{q}=0.025$, (b) $a m_{q}=0.01$.

doubled lattice. To save space, we show both masses from the wall source on the doubled lattice in Fig. 4. Full details of all the fits will be presented in a forthcoming more detailed paper. ${ }^{4}$

One promising aspect of the current calculation is the much improved chiral behavior of the $\pi_{2}$ and $\rho_{2}$ as compared with a previous calculation with stronger coupling. ${ }^{5}$ For instance, the current values for $\pi$ and $\pi_{2}$ with $a m_{q}=0.025$ are 0.415 (2) and $0.494(5)$, respectively. Previously, with $6 / g^{2}=5.4375$, we had $0.449(2)$ and $0.788(33)$. For the $\rho$ and $\rho_{2}$, we have $0.631(7)$ and $0.619(9)$, as compared with $0.856(106)$ and $0.787(77)$ previously.

In Fig. 5 we show our results on the Edinburgh plot. We have the two points we have calculated and an extrapolation to the chiral limit. To extrapolate, we have used a linear function for the $\rho$ and mucleon. Fo: the $\pi$, we use $m_{\pi}=a \sqrt{m_{q}}$ or $=a \sqrt{m_{q}}+b m_{q}$ as shown by a plain or dotted line, respectively. The points denoted by diamonds are from a previous calculation at stronger coupling and masses 0.10 , 0.05 and 0.025 . The octagons mark the experimental value and infinite quark mass value. The error bar going through the octagon marking the experimental value is the statistical error in the extrapolation. 


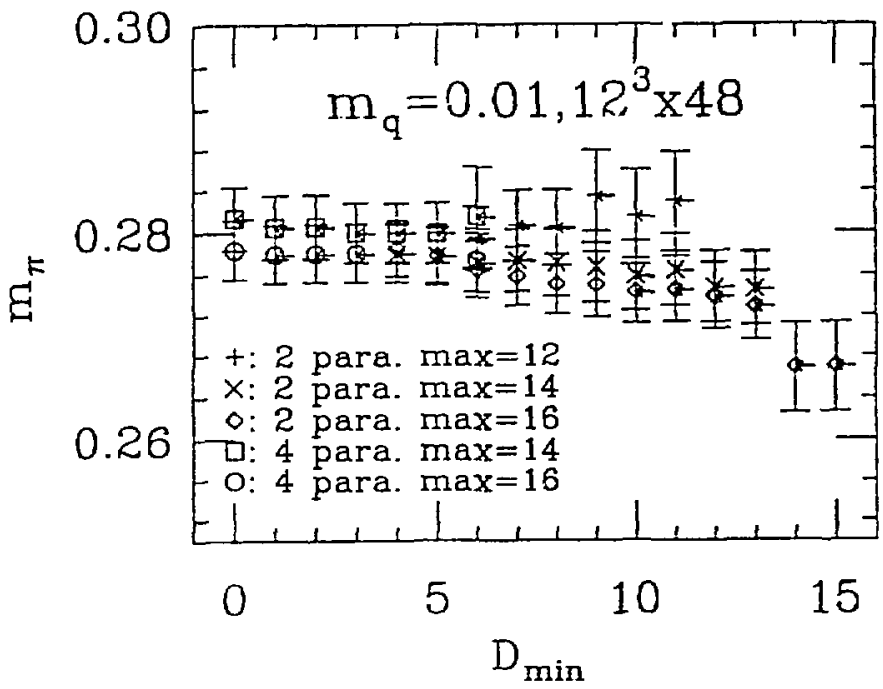

Figure 2. Pion mass for the quadrupled lattice as a function of the minimum distance from the source included in the fit for $\mathrm{cm}_{q}=0.01$.

\section{TABLE 1}

Summary of meson and nucleon masses.

\begin{tabular}{lll}
$a m_{q}$ & \multicolumn{1}{c}{0.025} & \multicolumn{1}{c}{0.010} \\
$\pi$ & $0.415(2)$ & $0.279(2)$ \\
$\pi_{2}$ & $0.494(5)$ & $0.351(8)$ \\
$\rho$ & $0.631(7)$ & $0.545(7)$ \\
$\rho_{2}$ & $0.619(9)$ & $0.539(10)$ \\
$N$ & $0.916(18)$ & $0.715(20)$
\end{tabular}

As noted above ${ }^{3}$, we have also calculated hadron masses with Wilson valence quarks on these configurations. The ratio $M_{N} / M_{\rho}$ with Wilson valence quarks is very different from that with staggered quarks. Adjusting the Wilson hopping parameter to give ihe same $\pi$ mass as for $a m_{q}=0.01$, the Wilson nucleon turns out to be at about 0.72 , in good agreement with the staggered result. However, the Wilson $\rho$ mass is around 0.4 , much lower than the staggered mass of 0.56 . There are clear finite lattice spacing effects, and work at weaker coupling is required before the results can be meaningfully compared with experiment.

\section{DISCLAIMER}

This report was prepared as an account of work sponsored by an agency of the United States Government. Neither the United States Government nor any agency thereof, nor any of their employees, makes any warranty, express or implied, or assumes any legal liability or responsibility for the accuracy, completeness, or usefulness of any information, apparatus, product, or process disclosed, or represents that its use would not infringe privately owned rights. Reference herein to any specific commercial product, process, or service by trade name, trademark, manufacturer, or otherwise does not necessarily constitute or imply its endorsement, recommendation, or favoring by the United States Gevernment or any agency thereof. The views and opinions of authors expressed herein do not necessarily state or reflect those of the United States Government or any agency thereof. 

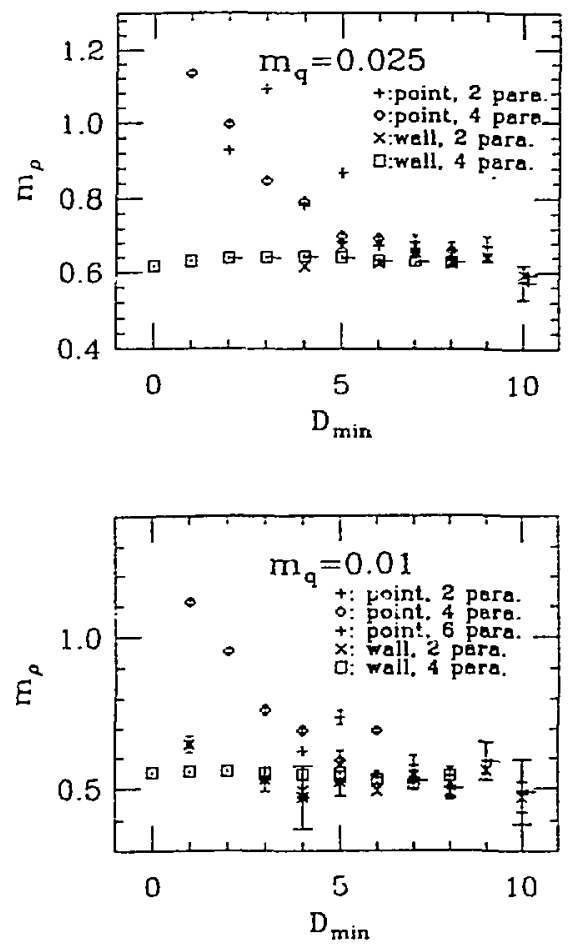

Figure 3. Same as Fig. 1, except for $\rho$.

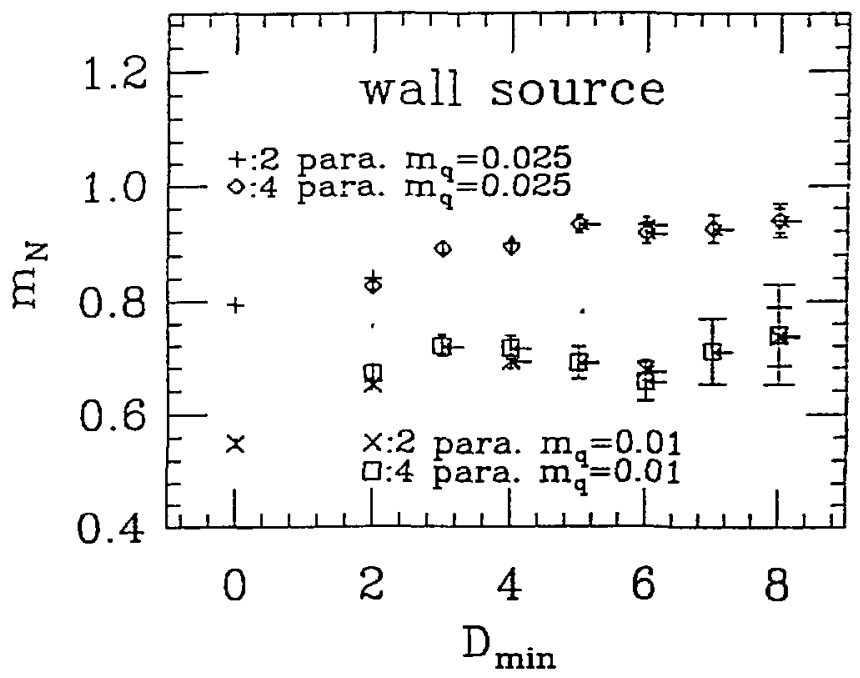

Figure 4. Nucleon riass for the doubled lattice. 


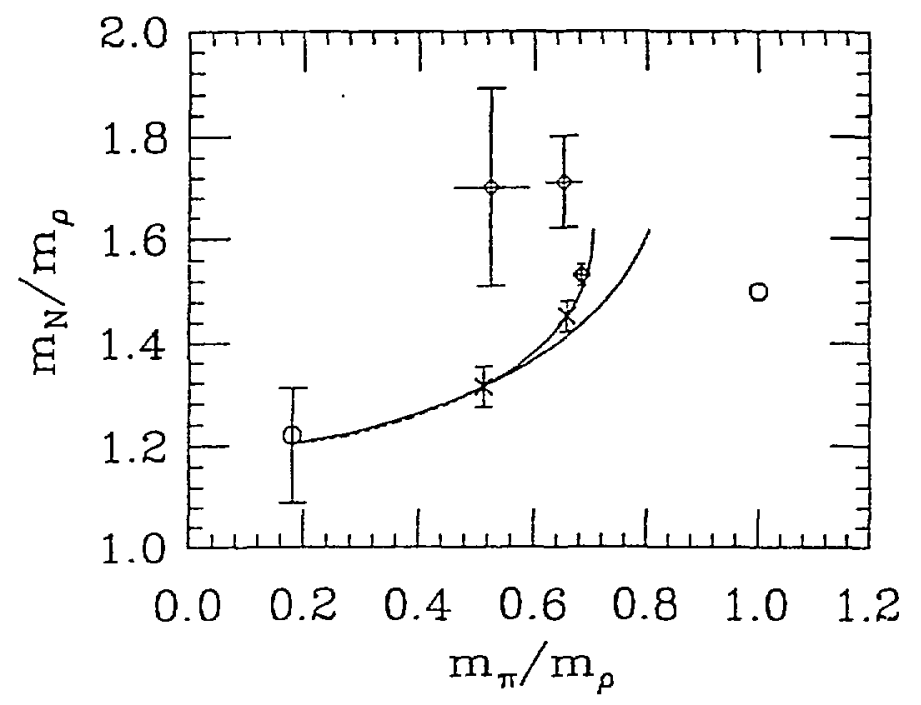

Figure 5. Edinburgh plot of current results and previous calculation at stronger coupling. 5

\section{REFERENCES}

1. See talks by C. Bernard et al., G. Kilcup et al. and K-F. Liu et al. in this volume.

2. S. Duane, Nucl. Phys. B257, 652 (1985) ; S. Duane and J. Kogut, Phys. Rev. Lett. 55, 2774 (1985); S. Gottlieb, W. Liu, R.L. Renken, R.L. Sugar and D. Toussaint, Phys. Rev. D 35, 2531 (1987).

3. Also in this volume.

4. S. Gottlieb, W. Liu, R.L. Renken, R.L. Sugar and D. Toussaint, Phys. Rev. D 38, 2245 (1988).

5. High Energy Monte Carlo Grand Challenge Collaboration, in preparation. 